\title{
TUCAN3G: CONSTRUCCIÓN DE UN MODELO DE NEGOCIO DE TELEFONÍA VIABLE PARA LAS ZONAS RURALES APARTADAS DE PERÚ Y COLOMBIA
}

\section{TUCAN3G: BUILDING A FEASIBLE TELEPHONE BUSINESS MODEL FOR REMOTE RURAL AREAS IN PERU AND COLOMBIA}

\author{
César Augusto Gómez Villamarín ${ }^{1}$ \\ Yury Yohana Castillo Molina ${ }^{2}$ \\ Omaira Calvo Giraldo ${ }^{3}$
}

\section{RESUMEN}

En el marco del proyecto Wireless technologies for isolated rural communities in developing countries based on cellular 3G femtocell deployments TUCAN3G, el cual busca una forma alternativa de llevar la tecnología celular 3G a comunidades rurales apartadas teniendo en cuenta los contextos de Perú y Colombia; el Centro Regional de Productividad e Innovación del Cauca CREPIC, participa dentro del paquete de trabajo denominado "Business Case" o Caso de Negocio que tiene como finalidad mostrar cual podría ser el posible modelo de negocio para la implementación de esta tecnología en estas comunidades. El componente "Business Case" está dividido en cuatro actividades principales, i) Análisis de Mercado, ii) Análisis desde de la Demanda, iii) Análisis desde la Oferta y iv) Diseño y verificación del modelo de negocio. Este artículo presentara algunos resultados producto del Análisis de mercado realizado en dos zonas rurales del departamento del Cauca en Colombia y algunas comunidades en la provincia de Loreto - Perú y el efecto de esta información en una de las primeras etapas de construcción de un modelo de negocio la segmentación de mercado.

Palabras clave: Modelo de negocio. Zonas rurales. Telefonía 3G.

\begin{abstract}
Under the project Wireless Technologies for Isolated Rural Communities in Developing Countries Based on Cellular 3G Femtocell Deployments TUCAN3G, which seeks an alternative way of bringing $3 \mathrm{G}$ cellular technology to remote rural communities taking into account the context of Peru and Colombia; the Regional Center for Productivity and Innovation of Cauca CREPIC is involved in the work package called "Business Case", which aims to show what could be the potential business model for the implementation of this technology in these communities. The "Business Case" component is divided into four main activities, i) Market analysis, ii) Demand analysis, iii) Supply analysis, and iv) Design and validation of the business model. This article will present some results from the market analysis in two rural areas of Cauca, Colombia, and some communities in the province of Loreto, Peru, and the effect of this information in one of the early stages of building a market segmentation business model.
\end{abstract}

Keywords: Business model. Rural áreas. 3G telephone.

\footnotetext{
${ }^{1}$ Centro Regional de Productividad e Innovación del Cauca (CREPIC), Colômbia. Carrera 10A, nº 1N-63, Barrio Modelo, Popayán Cauca-Colombia. E-mail: cagvillamarin@gmail.com

${ }^{2}$ Centro Regional de Productividad e Innovación del Cauca (CREPIC), Colômbia. Carrera 10A, nº 1N-63, Barrio Modelo, Popayán Cauca-Colombia. E-mail: yycastillo@gmail.com

${ }^{3}$ Centro Regional de Productividad e Innovación del Cauca (CREPIC), Colômbia. Carrera 10A, nº 1N-63, Barrio Modelo, Popayán Cauca-Colombia. E-mail: omairacalvog@gmail.com
} 


\section{Introducción}

Partiendo de la premisa de que la información y la comunicación son un factor clave del desarrollo humano, y considerando además la dificultad que existe en los países en vía de desarrollo para proveer a su población los medios necesarios para comunicarse, se empezó a ejecutar en al año 2013 el proyecto denominado "Wireless technologies for underdeveloped non-urban areas based on cellular $3 \mathrm{G}$ femto/relays deployments-TUCAN3G"4, el cual tiene como objetivo principal desarrollar sistemas de comunicación basados en la tecnología $3 \mathrm{G}$ que faciliten el acceso a los servicios de voz y datos a las comunidades de zonas rurales apartadas de países en desarrollo. Para cumplir este objetivo TUCAN 3G está estructurado en siete paquetes de trabajo que son: 1) administrativo y financiero, 2) requerimientos y especificaciones, 3) Caso de negocio, 4) optimización de las redes de acceso, 5) optimización de las redes de transporte, 6) plataforma de demostración y 7) diseminación y utilización del conocimiento; y para el desarrollo del proyecto fue conformada una alianza Universidad- Empresa- Estado de la que hacen parte 11 instituciones de España, Reino Unido, Perú, Grecia y Colombia.

TUCAN-3G no sólo trata de encontrar una solución tecnológicamente factible con el fin de prestar servicios $3 \mathrm{G}$ en las comunidades rurales, pues gran parte de su valor agregado radica en que el proyecto propondrá modelos de negocio sostenibles con el fin de que la solución tecnológica pueda ser atractiva para diversos públicos como son los operadores de telefonía celular, las agencias de cooperación internacional y fondos estatales de inversión de comunicaciones rurales, que tienen la facultad de implementar la tecnología en las áreas rurales remotas y de esta forma se logrará que los beneficios de los desarrollos tecnológicos lleguen a las comunidades que más los requieren.

\footnotetext{
${ }^{4}$ El proyecto TUCAN $3 G$ es financiado por el 7 mo programa marco de la Comunidad Europea. Este proyecto se inscribe en el objetivo ICT-b 03.10.2011 La intención de "poner en marcha un conjunto de proyectos de investigación dirigidos que traten el mismo equipo de la tecnología y modelo de negocio innovaciones, con especial énfasis en el desarrollo de tecnologías de bajo costo para promover el acceso y uso de las TIC. Es formulado y ejecutado por un consorcio de 11 instituciones de países de la Comunidad Europea y de países en desarrollo. Estas son: Universidad Politécnica de Catalunya, Universidad Rey Juan Carlos, Universidad Católica de Perú, Universidad del Cauca, FITEL, IP. Acces, Telefónica del Perú,

Fundación EHAS, Telefónica Internacional Wholesales Sevices, CREPIC y KINNO.
} 
La construcción de los modelos de negocio es liderada por la Fundación EHAS con la participación de Kinno Consulting, Universidad del Cauca, Telefónica del Perú, FITEL, IP acces y el Centro de productividad e innovación del Cauca-CREPIC, instituciones que han venido trabajando en la realización de estudios que han permitido conocer la realidad del mercado de la telefonía móvil en países en desarrollo. Estos estudios se han llevado a cabo en dos zonas rurales del departamento del Cauca en Colombia y en algunas comunidades radicadas en la zona de Loreto - Perú.

En este artículo se presentan los resultados obtenidos de los estudios de mercado, tanto en comunidades de Perú como de Colombia, y los posibles efectos que tendrá la información obtenida sobre la construcción del modelo de negocio para la tecnología desarrollada en el proyecto TUCAN 3G.

\section{Marco teórico}

\subsection{Modelo de negocios}

Desde finales de la década de 1990, ha habido una corriente de investigación viva proponiendo diferentes definiciones, listas de componentes, taxonomías, cambio en metodologias y modelos de evaluación de los modelos de negocio (BOUWMAN, 2003; EFACTORS, 2002; FABER et al., 2003; OSTERWALDER; PIGNEUR, 2002). Los temas tratados en la literatura modelo de negocio en esencia no son nuevos; los componentes de los modelos de negocio han sido reconocidos, por lo menos hasta cierto punto, en las estrategias de negocio y planificación de negocios durante décadas. Pero la necesidad de análisis y descripción del modelo de negocio se ha vuelto más explícita e inevitable. El concepto de modelo de negocio varía dependiendo de la fuente de las ideas y el área de aplicación. Es usado en áreas como la teoría tradicional de estrategia, la administración general, la literatura de la administración en información e innovación, en la ingenierita del negoció y principalmente se desarrolló con más fuerza en la industria de los negocios electrónicos (JANSEN et al., 2007).

Son variados los autores que han dado conceptos frente al modelo de negocio (SVEJENOVA et al., 2010; OSTERWALDER; PIGNEUR, 2009; ZOTT; AMIT, 2009; AL-DEBEI et al., 2008; MAGRETTA, 2002; PETROVIC et al., 2001; CHESBROUGH; ROSENBLOOM, 2001; LINDER; CANTRELL, 2000; TIMMERS, 1998; BRANDENBURGER; STUART, 1996) todos confluyen en una misma idea, que 
el modelo de negocio refleja los cuatro aspectos principales de la complejidad de una organización los clientes, la oferta, la infraestructura y la viabilidad financiera es decir que describen los fundamentos como una organización crea, captura y desarrolla valor.

Tradicionalmente, el negocio de una empresa se basa en la estrategia, que se refleja normalmente en los planes de negocio y las operaciones a nivel de productos específicos o grupos de productos. Sin embargo, a menudo existe un bache sustancial entre estos dos niveles. En particular, el rápido cambio tecnológico, junto con los factores sociales, organizacionales e individuales en el mercado global, hace que la gestión y la planificación de nuevas ideas de negocio sea complejo y desafiante (EFACTORS, 2002). Por lo tanto, hay una necesidad de una descripción sistemática de un modelo de negocio, los métodos y procesos para convertir las visiones estratégicas en medidas organizativas, responsabilidades y roles, información, materiales y flujos de dinero.

La virtud de los modelos de negocio es que consideran el contexto de los negocios desde un punto de vista conceptual, lo que hace que sea algo independiente de los procesos actuales y las restricciones de una organización. El modelo de negocio se puede ver como la implementación de la estrategia en un plan conceptual de la lógica de ganar dinero para las organizaciones (OSTERWALDER, 2004). En otras palabras, la visión de la empresa y su estrategia se traducen en propuestas de valor, relación con los clientes, las redes de valor, la tecnología, y los arreglos financieros

\subsection{Tic para el desarrollo}

El desarrollo como discurso ha tenido un amplio despliegue y como tal produce sentidos o imágenes de realidad, sentidos de verdad que detentan formas de poder y coacción (QUIJANO; TOBAR 2006). Así, se evidencia en el discurso del "Trato Justo" del presidente de los Estados Unidos en 1949 Harry Truman, el cual hace referencia a la división de los países, mencionando en primer lugar a aquellos que mediante su poderío económico, político y tecnológico les ha permitido alcanzar niveles de vida superiores y que por tanto se considerarían desde ese momento como países desarrollados. Por otro lado están los países que tenían economías muy débiles y deficitarias, sistemas políticos poco influyentes y casi ningún avance científico y tecnológico y por lo tanto su población vivía en condiciones precarias que están muy lejos de asimilarse a las 
condiciones de vida de las personas de los países desarrollados; estos países se conocen desde ese momento como países subdesarrollados.

A partir del discurso de Truman se empieza a posicionar en el mundo el desarrollo asociado a mejores condiciones de vida, capacidades científicas y tecnológicas y a crecimiento económico siendo este último el principal objetivo.

Como parte de los factores que contribuyen a este crecimiento económico están la ciencia, la tecnología y la innovación (CTeI) que se plantean como un mecanismo para alcanzar mayores niveles de desarrollo económico y social, por esta razón los países a través de diferentes políticas promueven cambios en el marco legal, institucional, financiero y organizacional (LICHA, 1997). Estas políticas se fundamentan teniendo en cuenta la importancia de la integración institucional que involucre a la academia, al sector productivo y al estado.

Al interior del enfoque de la CTI como factores clave para el progreso de las regiones y países, se encuentran las Tecnologías de la Información y la Comunicación TIC - como mecanismo que propician crecimiento económico al facilitar por ejemplo la educación de los habitantes. Sin embargo este no es un tema solo de infraestructura, sino que requiere que haya verdaderos procesos de apropiación de la tecnología, de lo contrario se convertiría en elementos de exclusión.

En varios países de América Latina una de las dificultades estructurales para alcanzar mejores niveles de desarrollo es la difícil topografía que impide la construcción de canales de transporte intra y entre regiones, así como la completa cobertura de servicios públicos básicos y el aprovechamiento de las tecnologías de la información y la comunicación. Estas restricciones actúan en desmedro de las condiciones sociales y económicas de las personas que habitan estas regiones desconectadas (ESCALAFÓN DE COMPETITIVIDAD DE LOS DEPARTAMENTOS DE COLOMBIA, 2009)

El proyecto TUCAN 3G busca desarrollar las herramientas tecnológicas que faciliten la comunicación entre las comunidades de áreas no urbanas apartadas y el mundo exterior sin importar las distancias, aportando con esto a disminuir las desigualdades sociales, dinamizar el crecimiento económico y el desarrollo social de aquellas comunidades de los países en desarrollo que al poder insertarse en la sociedad de la información aumentan sus capacidades de transformar y derribar las barreras 
que impiden el acceso a los beneficios provenientes de la participación social y productiva.

\section{Metodología}

El análisis del mercado es el primer componente del "Business Cases" en el proyecto TUCAN3G, permite comprender mejor, el diseño y la ejecución de todas las actividades necesarias para el desarrollo del modelo de negocio. Con este análisis se ofrece una panorámica clara, de los pros y los contras, del mercado potencial tanto en Perú como en Colombia, para los servicios que se desarrollará en el marco de TUCAN3G.

Con el fin de reunir y analizar información, indicaciones y pruebas en relación con el perfil de mercado de las regiones estudiadas, se han utilizado sistemas cualitativos y cuantitativos de herramientas, tales como cuestionarios y entrevistas semiestructuradas, siempre tomando en cuenta las características especiales que el grupo objetivo.

El análisis del mercado se realizó en tres etapas a saber:

- $\quad$ diseño del análisis de mercado;

- $\quad$ ejecución del análisis de mercado;

- $\quad$ análisis de los resultados.

\subsection{Etapa 1: diseño del análisis de mercado}

El primer paso en esta etapa fue la definición del alcance para el diseño del análisis de mercado en tres puntos: i) obtener información sobre los patrones de uso de los servicios de telecomunicaciones entre los grupos destinatarios, ii) para identificar oportunidades de mercado y las barreras que se utilizará para el desarrollo de los servicios de telecomunicaciones y TUCAN3G iii) entender el perfil de mercado de las zonas seleccionadas del proyecto. Paso seguido se definen los grupos objetivos tanto desde el lado de la oferta como desde la demanda.

Tabla 1 - Grupos objetivos principales 
- Los operadores de redes móviles $\mid$ Los usuarios privados

- Los fabricantes de equipos

- Los usuarios de negocio

- Sector Público

- Usuarios institucionales

Fuente: elaboración propia (2014).

Una vez definidos esto dos aspectos es momento de los métodos de recolección de datos y elección de los instrumentos para el análisis, como se había mencionado antes los dos métodos principales de recolección de datos que se han utilizado durante este análisis de mercado son los métodos cuantitativos y cualitativos. El uso de la combinación de estos métodos no sólo se ha traducido en un enfoque multidimensional para nuestros objetivos de investigación, sino que también nos dio la "libertad" de elegir y adaptar nuestras herramientas de investigación para la cada situación de los grupos objetivo.

Los métodos de recolección de datos cuantitativos se basan en un muestreo aleatorio y los instrumentos de recolección de datos estructurados que se adaptan a diversas experiencias en categorías de respuesta predeterminadas. Ellos producen resultados que son fáciles de resumir, comparar y generalizar. En este caso, se utilizaron encuestas con preguntas y cuestionarios de tipo cerrado para recopilar datos.

Por otro lado, los métodos cualitativos proporcionan información útil para comprender los procesos que subyacen a los resultados observados y pueden ser utilizados para mejorar la calidad de las evaluaciones cuantitativas basadas en encuestas, ayudando a generar hipótesis de evaluación mediante el fortalecimiento del diseño de cuestionarios de la encuesta y la ampliación o aclaración de los hallazgos de la evaluación cuantitativa. En general, estos métodos tienden a ser abiertas y tener protocolos menos estructurados.

Para cada grupo objetivo se estableció el método más adecuado para obtener la información necesaria: 
Tabla 2 - Herramientas de investigación para cada grupo objetivo

\begin{tabular}{l|l|l}
\multicolumn{1}{c|}{ Grupo } & \multicolumn{1}{c|}{ Tipo } & \multicolumn{1}{c}{ Herramienta } \\
\hline $\begin{array}{l}\text { Herramientas de Investigación para } \\
\text { el Sector Público (Funcionarios) }\end{array}$ & Cualitativa & Entrevistas abiertas \\
\hline $\begin{array}{l}\text { Herramientas de Investigación para } \\
\text { el Sector Público (Instituciones) }\end{array}$ & $\begin{array}{l}\text { Cualitativa y } \\
\text { cuantitativa }\end{array}$ & Entrevistas semi estructuradas \\
\hline $\begin{array}{l}\text { Herramientas de Investigación para } \\
\text { usuarios de negocios }\end{array}$ & Cualitativa & $\begin{array}{l}\text { Entrevistas semi estructuradas } \\
\text { para grupos focales }\end{array}$ \\
\hline $\begin{array}{l}\text { Herramientas de Investigación para } \\
\text { los usuarios privados }\end{array}$ & $\begin{array}{l}\text { Cualitativa y } \\
\text { cuantitativa }\end{array}$ & $\begin{array}{l}\text { Observadores, Cuestionarios } \\
\text { estructurados }\end{array}$ \\
\hline $\begin{array}{l}\text { Herramientas de Investigación para } \\
\text { los operadores }\end{array}$ & Cualitativa & Entrevistas semi estructuradas \\
\hline $\begin{array}{l}\text { Herramientas de Investigación para } \\
\text { los fabricantes }\end{array}$ & Cualitativa & Entrevistas semi estructuradas \\
\hline \multicolumn{2}{c}{ Fuente: elaboración propia (2014). }
\end{tabular}

La importancia de la personalización de algunas partes de la encuesta, de acuerdo con las necesidades del grupo objetivo de destino, era esencial. Además, la importancia de utilizar el idioma local, fue crucial para poder tener el mejor acercamiento a la población local. El desarrollo de las pruebas y localización de las herramientas metodológicas siguió tres fases:

i) Evaluación del grupo objetivo inicial: Se analizaron las preguntas y grupos objetivo.

ii) Traducción: Las traducciones en diferentes versiones fueron desarrolladas. Pruebas y ajustes iii) Revisión y ajuste de preguntas finales.

Por último se seleccionó la muestra para los grupos objetivo tanto para Perú como para Colombia.

\subsection{Etapa 2: Ejecución del análisis de mercado}

Los datos han sido recogidos por el uso de las herramientas ya mencionadas y para abarcar tanto a los usuarios y grupos objetivos de interés de la demanda y la oferta. Los datos cubren tanto la demanda como la oferta a los usuarios, así como los grupos objetivo. El número final de participantes se muestran en la siguiente tabla. 
Tabla 3 - Participantes en las actividades de investigación

\begin{tabular}{l|l|l|c}
\multicolumn{1}{c|}{ Grupo } & \multicolumn{1}{c|}{ Tipo } & Herramienta & $\begin{array}{c}\text { Participantes } \\
\text { finales }\end{array}$ \\
\hline $\begin{array}{l}\text { Herramientas de Investigación } \\
\text { para el Sector Público } \\
\text { (Funcionarios) }\end{array}$ & Cualitativa & $\begin{array}{l}\text { Entrevistas } \\
\text { abiertas }\end{array}$ & 4 participantes \\
\hline $\begin{array}{l}\text { Herramientas de Investigación } \\
\text { para el Sector Público } \\
\text { (Instituciones) }\end{array}$ & $\begin{array}{l}\text { Cualitativa y } \\
\text { cuantitativa }\end{array}$ & $\begin{array}{l}\text { Entrevistas semi - } \\
\text { estructuradas }\end{array}$ & 18 participantes \\
\hline $\begin{array}{l}\text { Herramientas de Investigación } \\
\text { para usuarios de negocios }\end{array}$ & Cualitativa & $\begin{array}{l}\text { Entrevistas semi - } \\
\text { estructuradas } \\
\text { para grupos } \\
\text { focales }\end{array}$ & 8 participantes \\
\hline $\begin{array}{l}\text { Herramientas de Investigación } \\
\text { para los usuarios privados }\end{array}$ & $\begin{array}{l}\text { Cualitativa y } \\
\text { cuantitativa }\end{array}$ & $\begin{array}{l}\text { Observadores, } \\
\text { Cuestionarios } \\
\text { estructurados }\end{array}$ & 419 participantes \\
\hline $\begin{array}{l}\text { Herramientas de Investigación } \\
\text { para los operadores }\end{array}$ & Cualitativa & $\begin{array}{l}\text { Entrevistas } \\
\text { semiestructuradas }\end{array}$ & 3 operadores \\
\hline $\begin{array}{l}\text { Herramientas de Investigación } \\
\text { para los fabricantes }\end{array}$ & Cualitativa & $\begin{array}{l}\text { Entrevistas } \\
\text { semiestructuradas }\end{array}$ & 5 compañías \\
\hline \multicolumn{1}{|c|}{ Fuente: elaboración propia (2014). } &
\end{tabular}

Los datos provenientes desde el lado de la demanda están relacionados con los usuarios privados, los usuarios empresariales y usuarios institucionales. Estos usuarios fueron encuestados varios lugares, con diferentes orígenes y ocupaciones que cubren tanto las zonas de Perú y Colombia, participaron en nuestra encuesta con el fin de entender mejor sus necesidades y exponer sus características y perfiles. Los datos recogidos de los usuarios privados que participaron en la encuesta fueron 419 personas, de los cuales 363 son de Perú y 56 de Colombia. Los datos recogidos de los usuarios de negocios o empresariales que participaron en la encuesta fueron 8 usuarios, de los cuales 5 son de Perú y 3 son de Colombia. Estos usuarios empresariales operan en los restaurantes y el transporte, mientras que algunos de ellos están operando en el campo de la fabricación artesanal y el turismo. Frente a los datos de los usuarios institucionales, 18 usuarios institucionales participaron en la encuesta, de los cuales 16 provienen de Perú y 2 provienen de Colombia. Los usuarios institucionales entrevistados operan en áreas como escuelas, comisarías de policía, centros de salud y centros comunitarios. 
Los datos provenientes desde el lado de la oferta se relacionan con las partes interesadas del sector público, operadores de redes móviles y fabricantes de equipos, procedentes principalmente de Perú o fuera de América del Sur. Estos actores y grupos de interés nos presentan el panorama general de los desafíos, los límites y las oportunidades relacionadas con el área examinada y cómo éstas podrían afectar a los servicios desarrollados a lo largo del proyecto TUCAN3G. Los funcionarios responsables del sector de las telecomunicaciones en las zonas rurales (en relación con el uso y el desarrollo de políticas en el campo de las telecomunicaciones) y los funcionarios responsables de la de otros sectores que desarrollan su actividad en zonas rurales fueron entrevistados sólo en el Perú con el fin de recoger todos los datos relevantes. En cuanto a los operadores de redes móviles, la metodología para la recopilación de información en relación con el mercado rural se basó en entrevistas semi-estructuradas con los principales operadores que trabajan en las zonas rurales de Perú. En primer lugar, se recogió una lista de los principales operadores en estas zonas de Asociación para el Fomento de la Infraestructura Nacional. De acuerdo con esta lista, solo 4 operadores tienen actividad en las áreas relacionadas y son: Telefónica del Perú (TdP), América Móvil Perú (Claro), Nextel del Perú y GILAT. Los fabricantes de equipos son críticos para el éxito global del proyecto. Para el alcance de TUCAN3G, una serie de todo el mundo las manufacturas han sido seleccionados para participar en nuestra encuesta. Estos fabricantes cubren toda la gama de equipos necesarios para la distribución de servicios, correspondiente a los que se desarrollarán dentro de las actividades TUCAN3G. Los fabricantes seleccionados para la entrevista fueron SiRRAN Communications Ltd, Altobridge, Sistelnetworks, iDirect e ip.access.

\subsection{Etapa 3: Análisis de los resultados}

La presentación de los resultados que se han elaborado a través de la investigación de todos los actores involucrados. Los temas más importantes resultantes de las encuestas se han destacado y se presentan, tanto para los actores institucionales de Perú y Colombia, y sus usuarios privados y empresariales. 


\section{Resultados del análisis de mercado}

\subsection{Lugar del estudio}

En caso colombiano se realizó el análisis en dos municipios del departamento del Cauca.

- $\quad$ El primer municipio que se visitó fue el municipio de Silvia a 59 kilómetros de la capital del departamento. El trabajo se realizó en una entrevista grupal con los socios de la "Asociación Productora y Comercializadora de Productos Acuícolas y Agrícolas APROPESCA”.

- $\quad$ También se visitó el municipio de Purace ubicado a 30 kilómetros de la capital del departamento, en este caso el levantamiento de información se realizó a diferentes habitantes de la cabecera municipal y veredas cercanas a Coconuco capital del municipio., además se entrevistó dos usuarios institucionales y dos usuarios privados como la Policía Nacional de Colombia, Empresa de Transporte Transtimbio, Puesto de Salud de Coconuco y la Hostería Coconuco - Comfandi.

Para el caso de Perú, se cuenta con una mayor número de usuarios e instituciones, ya que es el territorio peruano el pensado para para el despliegue de esta tecnología y con el apoyo del Fondo de Inversión en Telecomunicaciones quien realizo el trabajo de campo.

- Se visitaron cinco comunidades rurales aisladas ubicadas en el departamento de Loreto, el cual está ubicado al nororiente del territorio y está cubierto por bosques tropicales y atravesados por ríos gigantescos: i) San Juan de Armanayacu, ii) San Gabriel de Varadero, iii) Huitotos de Negro Urco, iv) Santa Clotilde y v) Tutapishco.

- $\quad$ Para los usuarios institucionales se trabajó con funcionarios responsables del sector de las telecomunicaciones en las zonas rurales (relacionado con el uso y el desarrollo de políticas en materia de telecomunicaciones): i) Fondo de Inversión en Telecomunicaciones (FITEL). ii) Organismo Supervisor de Inversión Privada en Telecomunicaciones (OSIPTEL), iii) Agencia para la Promoción de la Inversión Privada (PROINVERSIÓN), iv) Ministerio de Desarrollo e Inclusión Social, v) Ministerio de Salud, vi) Ministerio de Educación, vii) Ministerio del Interior. 
También con Los funcionarios responsables de las instituciones públicas con presencia en zonas rurales aisladas: i) Puesto de salud ii) escuela local, iii) comisaría de la localidad y vi) Autoridad de Gobierno Local de la localidad.

Figura 1 - Trabajo de campo en Colombia y Peru (2013)

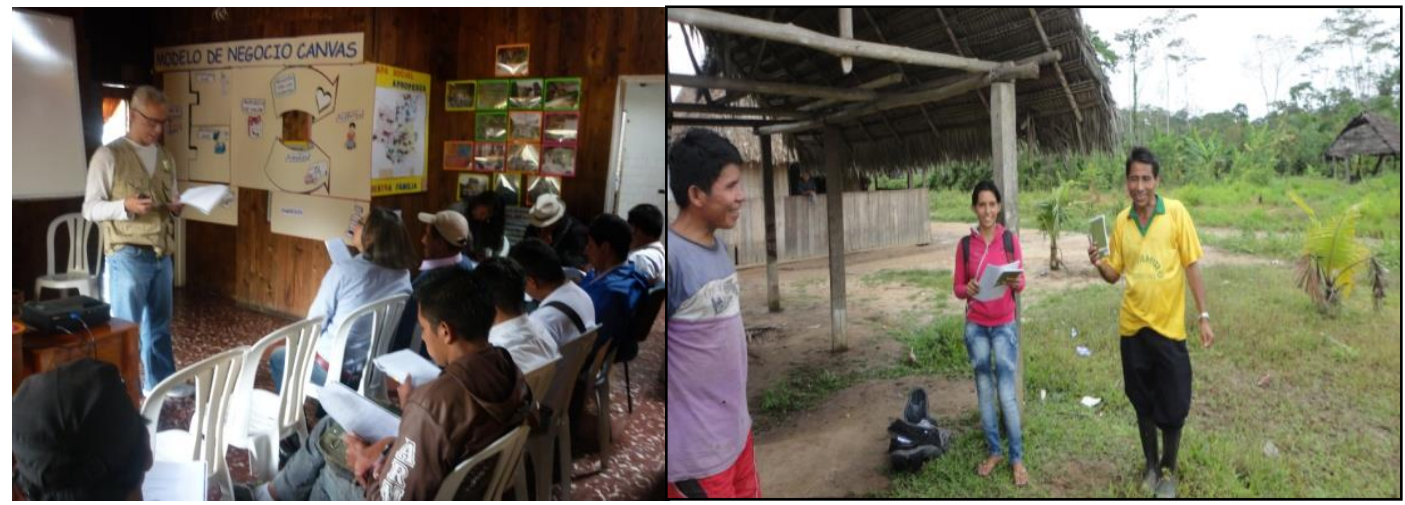

Fuente: dos autores (2014).

\subsection{Usuarios particulares}

Se realizó la encuesta a 419 personas y su rango de edad fue desde los 13 años de edad y hasta los 50 años de edad. En las comunidades visitadas predomina la utilización de los teléfonos celulares simples o de gama baja, con un $66 \%$ frente a otro medio como celulares de gama alta o Smartphone, computadores, sean estos de escritorio o personales o el uso de tablets. Los ingresos medios de estas comunidades tanto en Perú como en Colombia son muy similares rondando los US\$2.278 y son de carácter variable provenientes de su actividad como agricultores. Sin embargo los entrevistados estarían dispuestos a adquirir un teléfono de gama alta siempre y cuanto de les ofrezcan algunas facilidades como la compra a crédito en 12 meses o adquirir un equipo de características similares pero de bajo costo sin importarle la marca.

Figura 2 - Dispositivos utilizados por las comunidades de Colombia y Perú (2013)

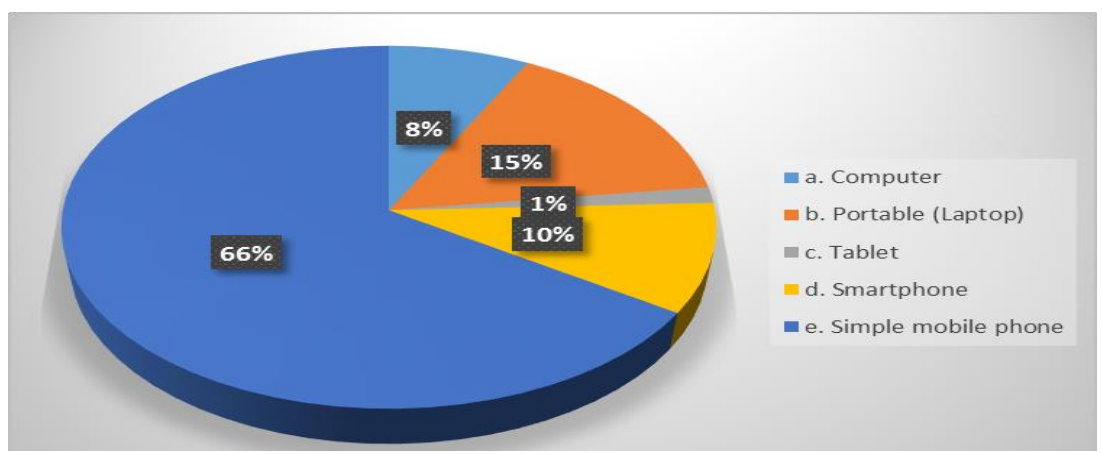

Fuente: elaboración propia (2014). 
Las comunidades encuestadas están divididas frente al uso del internet, ya que el $55 \%$ conoce de sus ventajas y $45 \%$ desconoce de ellas, esto debido a que en estas zonas su uso no es tan frecuente para evidenciar su importancia. Es por eso que los medios más usados para comunicarse con familiares que se han desplazado a otras zonas sea por trabajo o estudio son el teléfono celular o las cabinas de teléfonos públicos.

Con la implementación de la telefonía $3 \mathrm{G}$ en estas zonas daría acceso al mercado de las aplicaciones móviles la cual permite adquirir diferentes contenidos desde negocios, diversión, salud entre otros por su bajo costo. Los intereses manifestados se encuentran en la figura 3, siendo de mayor importancia las aplicaciones relacionadas con las noticias, los negocios, la educación y las redes sociales.

Figura 3 - Aplicaciones de interés para las comunidades de Perú y Colombia. 2013

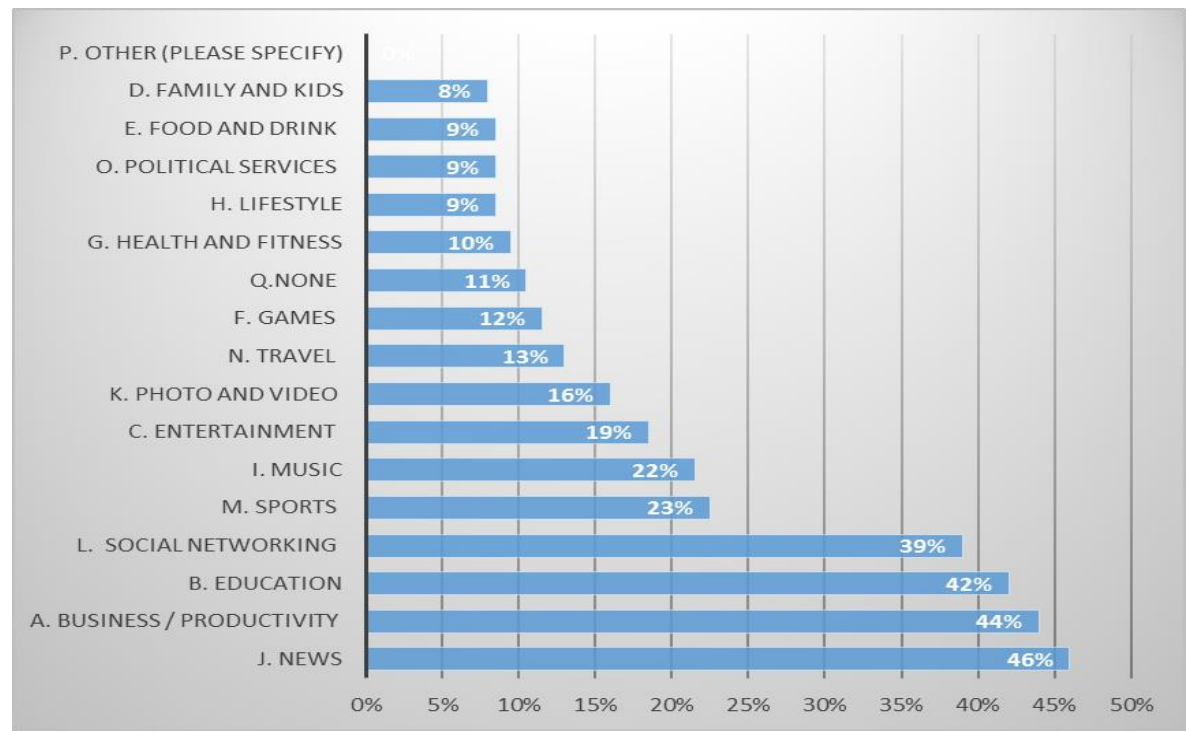

Fuente: elaboración propia (2014).

Un gran cambio que proveería este tipo de solución es que las personas tengan acceso tanto a internet como a telefonía en su casa, ya que lo normal es que estas comunidades se deban desplazar hacia las zonas de internet libre o teléfonos públicos para hacerlo. La Figura 4, muestra que algunas personas encuestadas respondieron que les gustaría tener acceso a internet tanto para actividades personales como de negocio en sus casas. 
Figura 4 - Uso y localización de los servicios de internet en Perú y Colombia (2013)

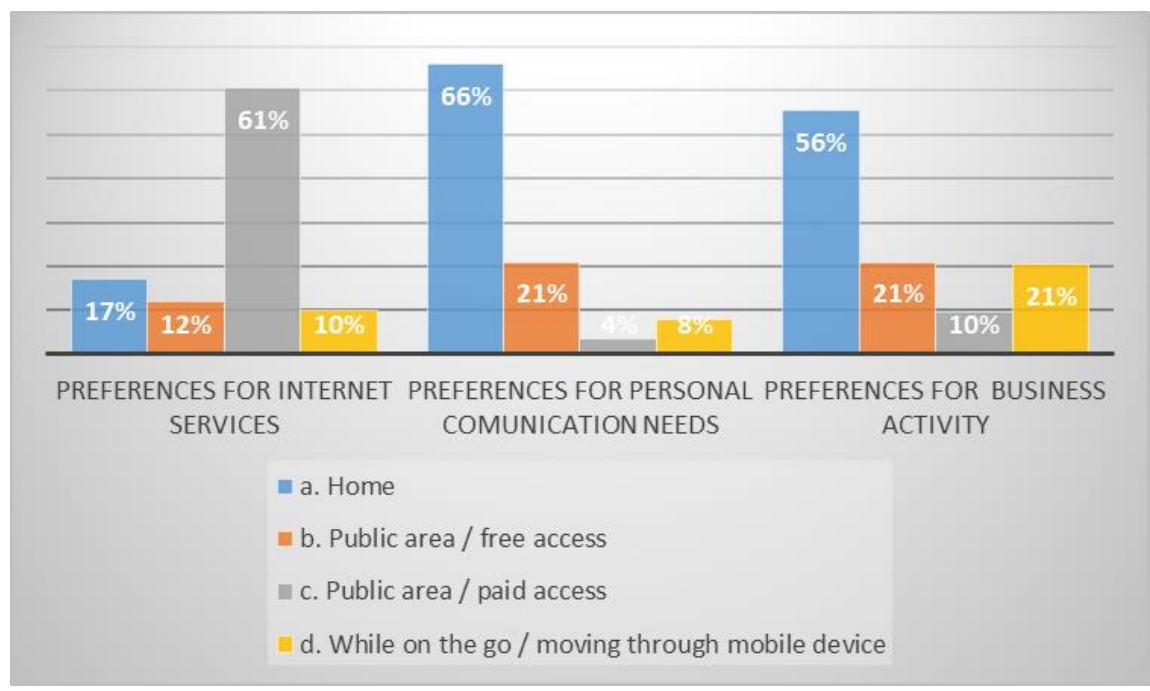

Fuente: elaboración propia (2014).

\subsection{Usuarios institucionales}

Los usuarios institucionales están convencidos de que el desarrollo de la tecnología 3G le permitirá a la gente a comunicarse mejor con los miembros de la familia, tener acceso a la información y las personas de trabajo y el mercado que puedan hacer transacciones rápidamente. Al igual que los centros de salud y educación entrevistados cree que la población local se benefició indirectamente de los servicios TUCAN3G, por la mejora de la calidad de los servicios ofrecidos. Los principales usos serian en los temas de correos intranet, en el sector salud para la implementación de historias clínicas electrónicas y nuevas formas de interactuar en la educación vía tecnologías de la información y las comunicaciones.

Los usuarios de negocios creen que la promoción de su negocio a través de Internet aumentaría sus ingresos ya que les permitiría comunicarse mejor con sus clientes. Especialmente los usuarios de negocios que operan en el sector turístico pueden mejorar su negocio con servicios en línea como de reserva, facturación y relaciones con los clientes. Los usuarios de negocios están dispuestos a comprar nuevos dispositivos que permiten el acceso a los servicios de Internet, así como, pagar una cuota por los servicios de Internet. Algo primordial es que el servicio prestado tiene que ser fiable y estable a pesar del clima u otras condiciones. 


\section{Conclusiones}

Por la difícil topografía, los altos costos que representa llevar servicios de telefonía móvil e internet a las zonas rurales apartadas de países como Colombia y Perú y la escasa concentración de población sumada a los altos niveles de pobreza; se requiere de desarrollos tecnológicos que brinden soluciones de bajo costo para que las familias que habitan estas zonas puedan acceder a estos servicios. Sin embargo, el éxito en la implementación y sostenibilidad de éstas depende de la voluntad de actores institucionales que estén comprometidos con mejorar las condiciones de vida de la población de bajos recursos que habita estas zonas.

Los estudios de mercado indican que existe una necesidad latente en las comunidades de las zonas rurales apartadas de acceder fácilmente a los servicios de la telefonía móvil. Pero aunque existe la necesidad, estas personas no cuentan con la capacidad económica necesaria para pagar la cantidad de dinero que pueden requerir los operadores para que el negocio de llevar telefonía móvil hasta estas zonas sea rentable. Por este motivo, el modelo de negocio que se perfile en esta investigación debe incluir como socio clave al estado porque es deber de este mejorar los niveles de cobertura en servicio básicos en todas las regiones del país. Así mismo es importante que se involucre como otro socio clave a la comunidad que puede colaborar con el mantenimiento y seguridad de la infraestructura física y de ésta forma contribuir a reducir los costos de implementar tecnologías como la desarrollada en TUCAN 3G.

Dadas las limitaciones económicas de los usuarios finales para acceder a los servicios de voz y datos, es importante que el modelo de negocios considere la opción de incluir en el portafolio servicios básicos pero de buena calidad para atender las necesidades de la población.

Es importante que se desplieguen estrategias de apropiación de conocimiento para que los usuarios finales de la tecnología conozcan los benéficos de ésta y para puedan hacer uso eficiente de los servicios que se puede poner a disposición de ellos, por ejemplo incluyendo el internet en el mejoramiento los procesos educativos. 


\section{Agradecimientos}

Sinceros agradecimientos al proyecto Wireless technologies for isolated rural communities in developing countries based on cellular 3G femtocell deployments TUCAN3G y al consorcio que ejecuta esta propuesta.

\section{REFERENCIAS}

AL-DEBEI, M. M.; EL-HADDADEH, R.; AVISON, D. Defining the Business Model in the New World of Digital Business. Proceedings of the Fourteenth Americas Conference on Information Systems, p. 1-11, 2008.

BOUWMAN, H. State of the art on business models, B4U/D3.2. Telematica. Instituut, TU Delft, TNO, KPN research, p.42, 2003.

BRANDENBURGER, A. M.; STUART, H. Value-based Business Strategy. J. Econom. \& Management Strategy, v. 5, n. 1, p. 5-25, 1996.

CHESBROUGH, H.; ROSENBLOOM, R. S. The role of the business model in capturing value from innovation: Evidence from Xerox Corporation's technology spinoff companies. Industrial and Corporate Change, v. 11, n. 3, p. 529-555, 2002.

E FACTORS. WP3: E-business model roadmap. Deliverable 3.1. of Information Systems, 2002

FABER, E. et al. Designing business models for mobile ICT services. Proceedings of the 16 $^{\text {th }}$ Bled Electronic Commerce Conference, 2003.

LICHA, I. Las Nuevas Políticas Cientificas Para La Competitividad. El Caso Latinoamericano. In: SUT, J. Innovación y desarrollo en América Latina. Venezuela: Consejo Latinoamericano de Ciencias Sociales - CLACSO, 1997. p. 135150.

LINDER, J.; CANTRELL, S. Changing Business Models: Surveying the Landscape, Accenture Institute for strategic Change, 2000.

MAGRETTA, J. Why business models matter. Harvard Business Review, v. 80, n. 5, p. 86-93, 2002.

OSTERWALDER, A. The business model ontology - A proposition in a design 
science approach. Tese (Doutorado) - University of Lausanne, Lausanne, 2004.

OSTERWALDER, A.; PIGNEUR, Y. An e-business model ontology for modelling ebusiness. Proceedings of the $\mathbf{1 5}^{\text {th }}$ Bled Conference on E- Commerce, Bled, Slovenia, 2002.

OSTERWALDER, A.; PIGNEUR, Y. Business model generation. 2009. Disponible en:

<http://www.businessmodelgeneration.com/downloads/businessmodelgeneration_previ ew.pdf>.

PETROVIC, O.; KITTL, C.; TEKSTEN, D. Developing business models for e-business. Proceedings of the International conference on Electronic Commerce, 2001.

SVEJENOVA, S.; PLANELLAS, M.; VIVES, L. An individual business model in the making: a Chef's quest for creative freedom. Long Range Planning, v. 43, p. 408-430, 2010.

TIMMERS, P. Business models for electronic markets. Electronic Markets, v. 8, n. 2, p. 3-8, 1998.

QUIJANO, T. B. O. Desarrollo, sentidos y diferencia. In: Discursos y prácticas del desarrollo globalocal. Universidad del Cauca, 2007. p. 9.

ZOTT, C.; AMIT, R. Innovación del modelo de negocio: creación de valor en tiempos de cambio. Universia Business Review, v. 23, p. 108-121, 2009.

Recebimento dos originais: 07/05/2015

Aceitação para publicação: 26/08/2015 